\title{
Concepts of inhomogeneous illumination of area-based light modulators for high-resolution headlamps
}

\section{Yang Li, Marvin Knöchelmann, Roland Lachmayer}

Yang Li, Marvin Knöchelmann, Roland Lachmayer, "Concepts of inhomogeneous illumination of area-based light modulators for high-resolution headlamps," Proc. SPIE 10932, Emerging Digital Micromirror Device Based Systems and Applications XI, 109320P (4 March 2019); doi: $10.1117 / 12.2508620$

SPIE. Event: SPIE OPTO, 2019, San Francisco, California, United States 


\title{
Concepts of inhomogeneous illumination of area-based light modulators for high-resolution headlamps
}

\author{
Yang Li, Marvin Knöchelmann, and Roland Lachmayer \\ Institute of Product Development, Leibniz University Hannover, Welfengarten 1A, 30167 \\ Hannover, Germany
}

\begin{abstract}
Adaptive headlamps with innovative lighting functionalities can increase traffic safety. Subtractive light modulators such as Digital-Micromirror-Devices (DMD), liquid crystal displays (LCD) or liquid crystal on silicon devices (LCoS) are considered to be used as an implementation with a high resolution. In order to realize the regulated light distribution as well as to improve the optical efficiency and on-road projection quality of such headlamp systems, an inhomogeneous illumination on the modulator and whereafter low distortion projection optics are considered. In this paper we present simulation results of an optical concept of inhomogeneous illumination for headlamps.
\end{abstract}

Keywords: High-resolution headlamp, Pixel-light headlamp, DMD, LCoS, LCD

\section{INTRODUCTION}

Though various projection technologies are integrated in vehicle headlamp systems for enhancing the driving experience and road safety, the requirements between a headlamp and a projector are dissimilar. Projectors demand an uniform light distribution on the target screen for a enjoyable watching experience, nevertheless vehicle front illumination requires a more concentrative pattern in front of the vehicle on the road surface, namely inhomogeneous distribution.

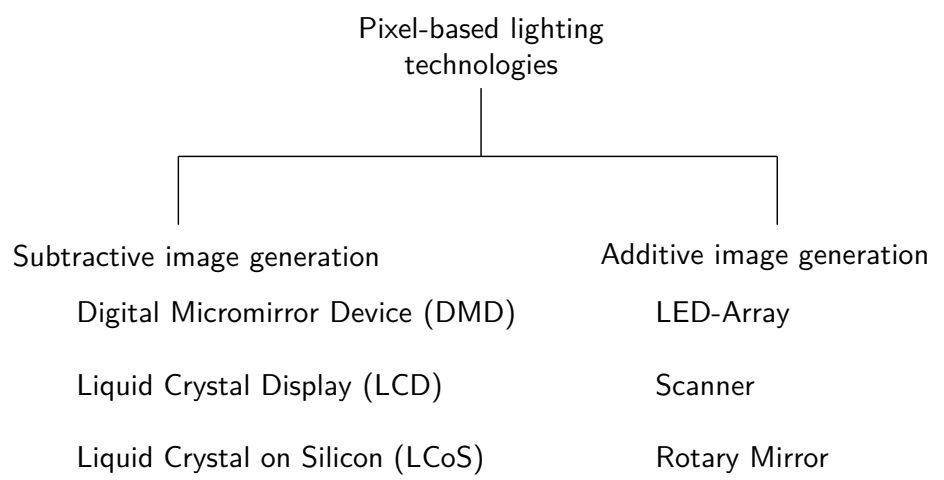

Figure 1. Classification of modulator technologies in additive and subtractive modulation ${ }^{1}$

For the technical implementation of high-resolution headlamps different technologies come into question, which can be differentiated by additive and subtractive modulators (Figure 1). Additive image generation approach actualizes the dynamic projection pattern by switching on and off, or dimming the light sources in a rapid frequency. The light source lightens only when it is needed, so that a high system efficiency can be achieved. A typical example of this approach to project on-road content is the laser scanner, which consists of RGB laser diodes and a scanning mirror. An operational prototype of a laser scanner is presented by Kloppenburg. ${ }^{2}$ In subtractive systems the light source illuminates an image modulator continuously. To form an image, light is deflected or absorbed to turn off individual pixels. Because of this working principle the system efficiency of

Further author information:

Yang Li: E-mail: y.li@ipeg.uni-hannover.de, Telephone: +49 5117622723

Emerging Digital Micromirror Device Based Systems and Applications XI, edited by

Michael R. Douglass, John Ehmke, Benjamin L. Lee, Proc. of SPIE Vol. 10932, 109320P

(C) 2019 SPIE $\cdot$ CCC code: $0277-786$ X/19/\$18 $\cdot$ doi: $10.1117 / 12.2508620$

Proc. of SPIE Vol. 10932 109320P-1 
headlamps with subtractive image generation is theoretically lower than with additive image generation. The focus of this paper are subtractive modulators, as these can generate a very high resolution, but also present special challenges due to the method of image generation. ${ }^{3}$ The three challenges in implementing a high resolution headlamp to generate complete light distributions with a subtractive image modulator are:

- Adjust the aspect ratio

- Create a central hotspot

- Increase system efficiency

With radial distorting projection optics the mentioned challenges can be solved. ${ }^{4}$ Due to the strong distortion of the optics, the lenses must have small radii of curvature. This leads to a pronounced chromatic aberration and to the fact that the sharpness and the contrast decrease significantly.

One possible solution to this problem is to inhomogeneously illuminate the image modulator. Due to this pre-distribution of the light, the subsequent distortion of the projection optics can be significantly lower. This can be expected to improve important features of the system such as contrast and sharpness.

\section{REQUIREMENTS}

In Figure 2 the measuring screen and relevant test point locations of the right-handed traffic for a high beam distribution are shown. In Table 1 the illumination requirements of these points using class $\mathrm{D}$ vehicles as an example according to regulations from $\mathrm{UNECE}^{5}$ are listed. It can be seen that the minimum illuminance value at point 2 which locates at around $\pm 3^{\circ}$ along the horizontal direction in front of the vehicle is triple of the value at point 3 locating at $\pm 6^{\circ}$. Considering the regulations for low beam distribution and energy saving reasons as well, a central hotspot leading to a better illumination than peripheric areas is expected.

Table 1. Regulated illumination of the test points in figure 3 for class D vehicles according to $\mathrm{UNECE}^{5}$

\begin{tabular}{c|c|c|c|c|c|c|c}
\hline Test Point Number & 1 & 2 & 3 & 4 & 5 & 6 & 7 \\
\hline Min Illumination (lx) & 49.6 & 19.2 & 6.4 & 3.84 & 1.28 & 1.92 & - \\
\hline Max Illumination (lx) & - & - & - & - & - & - & 54 \\
\hline
\end{tabular}

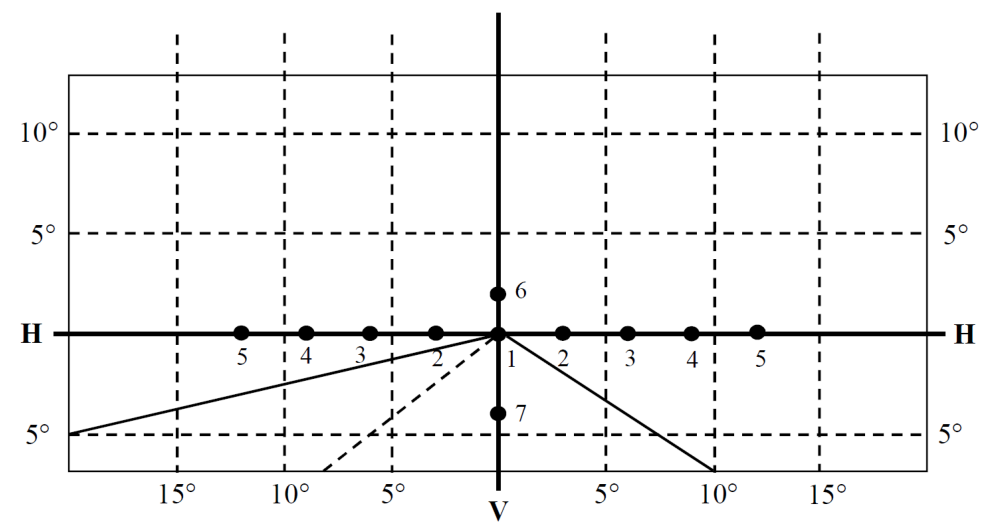

Figure 2. Measure plane and testing points of the primary driving beam for a vehicle headlamp. ${ }^{5}$

Real light distributions are based on the described UNECE regulation, but the actual illuminance clearly exceeds the requirements. Figure 3 shows an example of a real high beam distribution of a series headlight. In the center, a maximum illuminance of $156 \mathrm{~lx}$ is reached. The total luminous flux of the distribution is $1440 \mathrm{~lm}$. High-resolution lighting functions such as a glare-free high beam or a masking light use the central area of the light distribution, which is shown in the figure with a black frame. In the marked area of $\pm 15^{\circ}$ horizontally and 


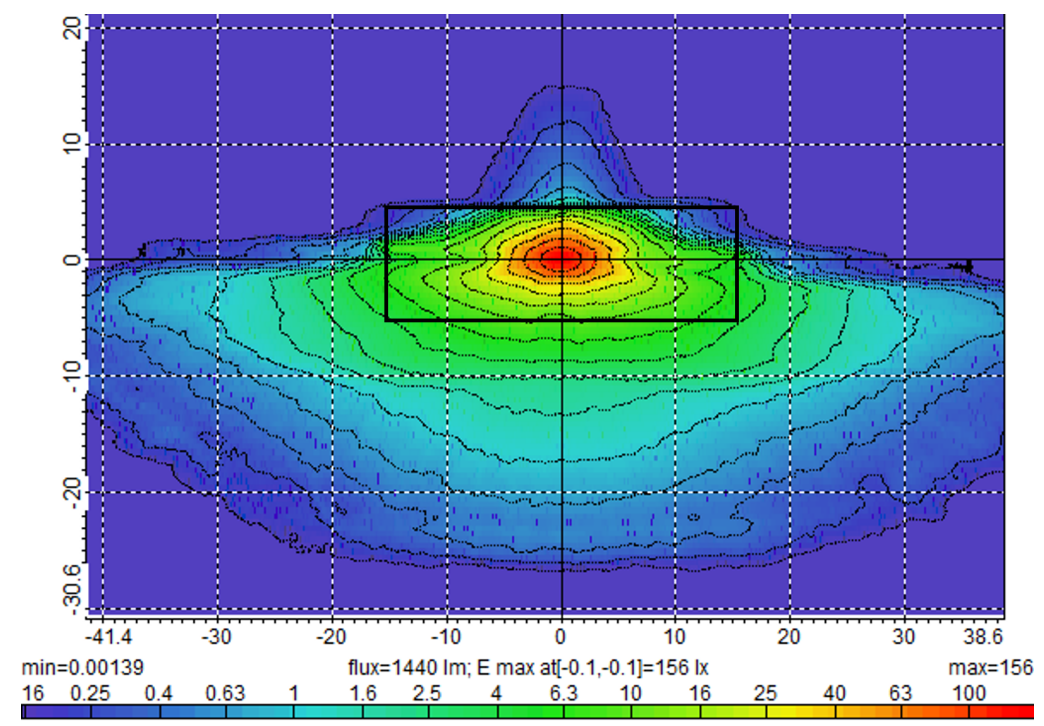

Figure 3. High-beam distribution of a series headlamp.

$\pm 5^{\circ}$ vertically, $70 \%$ of the total luminous flux is emitted. In order to generate a complete light distribution, an additional module for the basic light distribution is necessary. The concept of inhomogeneous illumination of a light modulator presented in this article is intended to generate the partial area of the light distribution shown in Figure 3. In addition to the absolute maximum a digital cornering light should be implemented, which is benefit for drivers to previously illuminate a corner before turning.

\section{SYSTEM EFFICIENCY}

Since the system efficiency of subtractive modulators is closely related to the optical system and the generated light distribution, it is considered more closely. The system efficiency of high-resolution headlamps depends largely on two factors:

Optical efficiency The number of lenses, the numerical aperture, the material used, coatings and radii of curvature directly affect the optical efficiency of the system.

Utilization factor The system efficiency is also determined by how well the optical system is adapted to the currently generated light distribution, which is described by the so-called utilization factor. ${ }^{6}$

For system efficiency analysis, consider the system shown in Figure 4. The light source converts electrical power $P_{L S, e l}$ into the visible luminous flux $\Phi_{L S}$. The illumination optics collects this light and illuminates the modulator with an efficiency of $\eta_{I O}$. The efficiency of the modulator $\eta_{M}$ depends on the chosen technology. The electrical power of subtractive modulators $P_{M, e l}$ is small compared to the power of the light source. Depending on the number and coating of the lenses and the distortion, the optical efficiency $\eta_{P O}$ of the projection optics results. The luminous flux behind the projection optics $\Phi_{P O}$ corresponds to the luminous flux of the currently generated light distribution in the traffic space.

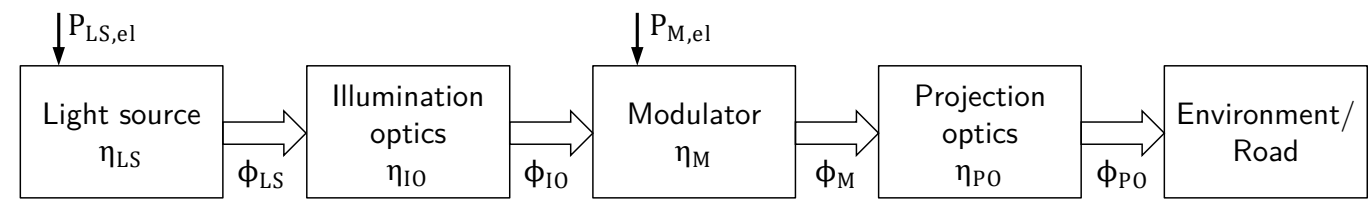

Figure 4. Energy flow through the optical basic system with subtractive modulator. 
Additive modulators, e.g. a LED-array, combine the light source and the image modulator in one single component. Thus, a separate the illumination optics is not necessary. The efficiency $\eta_{A d d}$ of a high-resolution headlight with additive image generation therefore results in:

$$
\eta_{A d d}=\eta_{M} \cdot \eta_{P O}=\frac{\Phi_{P O}}{P_{M, e l}}
$$

Subtractive modulators, e.g. DMD, LCoS, or LCD, require more optical components due to the extra lighting path, which theoretically reduces system efficiency compared to additive modulators. A second disadvantage of subtractive modulators in terms of system efficiency is that in the generation of a particular light distribution, individual pixels of the image modulator must be dimmed or turned off. The light source continues to illuminate the modulator with an unchanged luminous flux, resulting in reduced system efficiency. This effect is described with the utilization factor $K_{F}$, which can be determined for a specific lighting function in combination with an optical system. ${ }^{6}$ The efficiency $\eta_{\text {Sub }}$ therefore results from the product of the efficiency chain from Figure 4 and the utilization factor to:

$$
\eta_{S u b}=\eta_{L S} \cdot \eta_{I O} \cdot \eta_{M} \cdot \eta_{P O} \cdot K_{F}=\frac{\Phi_{P O}}{P_{L S, e l}}
$$

In order to calculate the utilization factor, the light function to be examined must be described discretely. For this, the luminous flux matrix $A_{\phi}$ can be set up:

$$
A_{\phi}=\left[\begin{array}{rrr}
\phi_{F, 11} & \cdots & \phi_{F, 1 n} \\
\vdots & \ddots & \vdots \\
\phi_{F, m 1} & \cdots & \phi_{F, m n}
\end{array}\right]
$$

The luminous flux of a light function with the solid angle at the angular position $i, j$ is $\phi_{F, i j}$. The utilization $K_{F}$ factor can then be determined to:

$$
K_{F}=\frac{\sum_{i=1}^{m} \sum_{j=1}^{n} \phi_{F, i j}}{\max \left(A_{\phi}\right) \cdot m \cdot n}
$$

In order to achieve the required illuminance (Figure 3) in the marked angle range, a luminous flux of $9142 \mathrm{~lm}$ is necessary for homogeneous illumination of the modulator and for non-distorting projection optics. This value results from the spanned area of the angle range in $25 \mathrm{~m}$ distance multiplied by the maximum illuminance of $156 \mathrm{~lx}$. The utilization factor in this case is $11 \%$. The concept of inhomogeneous lighting is intended to show that the utilization factor can be significantly increased.

\section{CONCEPTS FOR INHOMOGENEOUS ILLUMINATION}

A light modulator can easily generate the light distribution with a hotspot by displaying grey scales, but the optical efficiency is fairly low due to the forementioned subtractive approach. Thus there are several concepts to generate a hotspot in area-based high resolution headlamp systems have been brought up. Günther et al. preset an approach which uses an ellipsoid reflector with illumination optics to realize this required distribution pattern on the DMD surface, ${ }^{7}$ Bhakta et al. present another concept using all transparent optics with a white LED to produce a similar pattern. ${ }^{8}$ The design of the components using these approaches differ from diverse light sources. In contrast to these pre-shaping concepts, in previous work a pincushion distortion projection optic was presented to centralize the out put light in the central area, which allows a more or less homogeneous illumination on the DMD chip. ${ }^{9}$ This method is flexible to different light sources and display chips, but requires an algorithm to calibrate the projection content, and brings side effects like high blur and strong chromatic aberration in some cases. 
A micro lens array (MLA) is universally used as homogenizer in projection systems in front of a modulator to realize a uniform distribution. A MLA composed of multi-lenslets so that it can divide a parallel input light into several sub-beams. After the MLA a relay lens is used to collect and superpose these sub-beams onto the modulator's display area. For the homogenizing application the focus lengths of all lenslets on a MLA are the same. Furthermore, these lenslets have the same aspect ratio as the light modulator, thus each sub-beam can reach the same form and dimension as the modulator's functional surface, then targets to the same area with the help of the relay lens as showed in Figure 5.

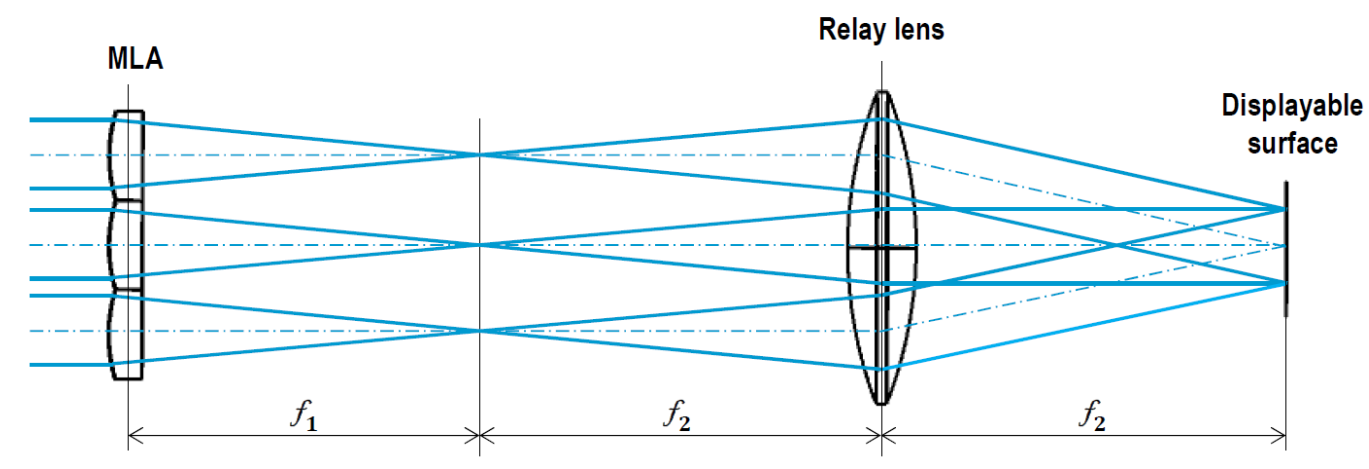

Figure 5. A homogenizer concept using a MLA and a relay lens, $f_{1}$ is the focus length of each lenslet on the MLA, $f_{2}$ is the focus length of the relay lens.

However, a MLA can be designed not only as a homogenizer. The focus length of each single lenslet on the MLA is the key factor of the illumination area of its sub-beam on the display surface. A longer focus length in the system results a more centralized distribution area, and this method can be used for the central hotspot generation. An example based on the model in Figure 5 is illustrated in Figure 6.

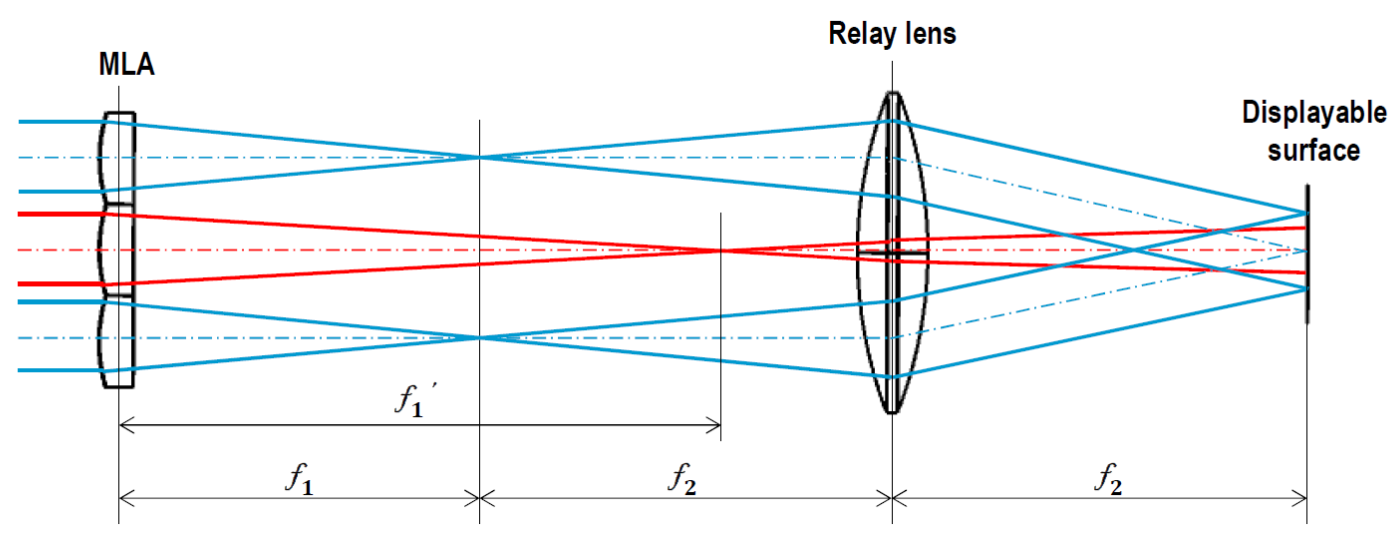

Figure 6. An inhomogeneous concept using an MLA and a relay lens, $f_{1}$ and $f_{2}$ are the same as in Figure 5, and $f_{1}^{\prime}$ is the focus length of the the central lenslet on the MLA.

Compared to the MLA in Figure 5, the MLA for a hotspot generation in Figure 6 is composed of lenslets which have different focus lengths. The upper and lower lenslets with the focus length of $f_{1}$ are used to illuminate the whole modulator, while the central lenslet focuses the light more in the center with the relay lens. In such a manner a basic inhomogeneous illumination pattern can be realised on the modulator. When a MLA with more lenslets of various focus lengths is used, different illumination levels for specific areas on the modulator surface are possible. 
In addition, this approach has an issue. The concentrated light density of the hotspot intends to bring an intense heat load on a modulator's surface. This might cause negative impacts on the performance of the modulator, in the worst case, leads permanent damage to them. Therefore proper thermal dissipations must be considered for the application of this method to release the heat pressure. Besides, the design of such special MLAs might cause difficulties to the manufacture. However, as additive manufacturing of transparent optics has been continuously developing, it opens the possibility to precisely manufacture different individual MLAs by requests from various modules of high-resolution headlamps in the future.

The use of this MLA requires an as parallel as possible input light. For current widely used light sources such as Xenon bulbs, LEDs and even Laser diodes, a nearly parallel light can be achieved by collimators using reflectors and collimation lenses in many cases. Once such a MLA is used, other optics for hotspot generation are no longer needed, except for projecting optics to shape the aspect ratio of the illumination pattern for headlamps after the modulator.

\section{MODELS AND RESULTS (SIMULATION)}

Based on the working principle and design method mentioned above, a simulation model for a high resolution headlamp is created in Zemax OpticStudio. An area based light modulator with a typical size of $12.29 \mathrm{~mm} \times$ $7.37 \mathrm{~mm}$ is used for generating the pixel light. For simplifying the simulation system, the modulator is set perpendicularly to the optical axis, thus can be an LCD or an LCoS. However a DMD chip is also possible when a Total Internal Reflection prism (TIR) is used or the DMD is set obliquely to the optical axis. The light source of the simulated optical system is a parallel incident light. Even though a parallel light is too ideal for a realistic optical system, in many cases it can be achieved by using appropriate optical components with an acceptable tolerance when using LEDs or laser diodes as light sources. After this light source is the MLA located. The MLA has $10 \times 6$ lenslets, each of them has the same aspect ratio as the modulator of $5: 3$. An aspherical lens is used as the relay lens behind the MLA to gather and project the light on the target area. In this way an inhomogeneous light distribution with a central hotspot can be achieved on the modulator. Considering the actual performance of the liquid crystal devices and the DMD chips, the MLA and the relay lens group is designed to produce the light on the modulator's surface with an incident cone angle of $12^{\circ}$, with which the dimension and the performance of any such a headlamp system composed of a liquid crystal device or a DMD could reach a balance. ${ }^{10,11}$ The optical efficiency with PMMA optical components is $78.2 \%$ according to the simulation. The result of this light shaping is showed in Figure 7.

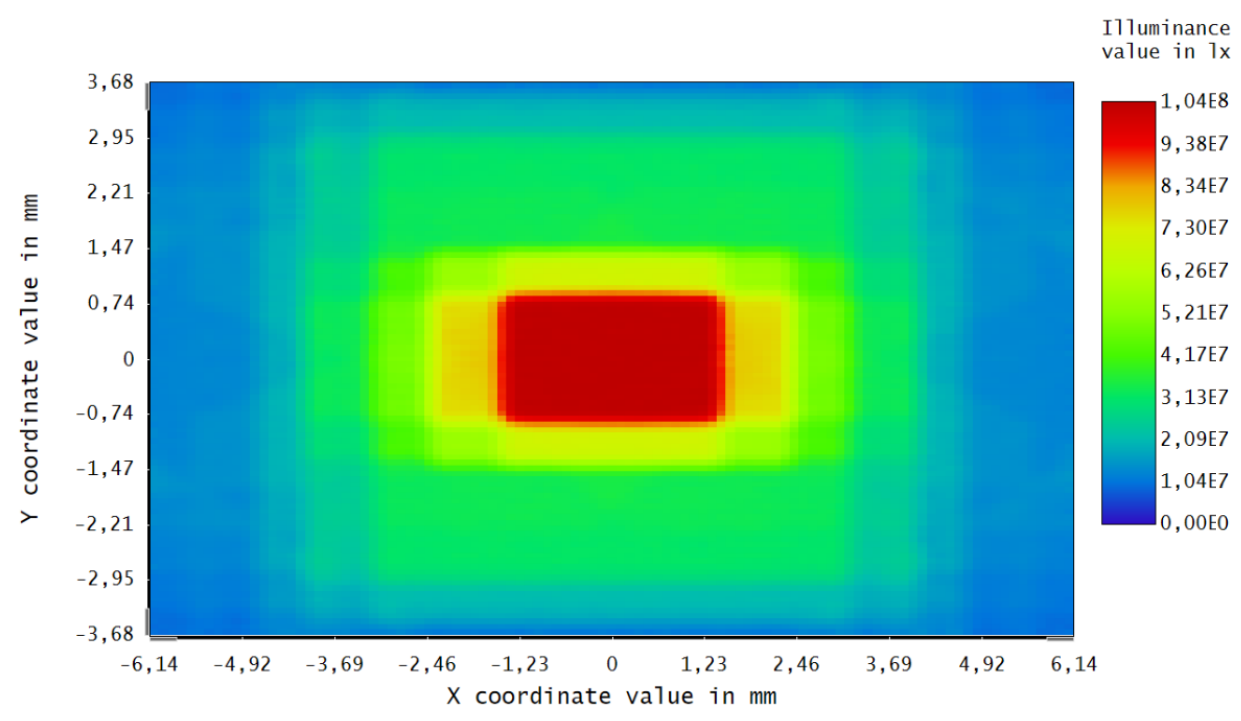

Figure 7. Simulated light distribution on the light modulator's surface. 
After the light is projected on the modulator's surface, projection optics which intend to change the aspect ratio of the illumination pattern are used. These optics broaden the output light in horizontal direction and compress it in vertical direction, consequently the required headlamp's distribution aspect ratio of $\pm 15^{\circ} \times \pm 5^{\circ}$ is realized. In the last place a detector locating at $25 \mathrm{~m}$ distance in front of the projection optics is utilized to observe the whole optical system's performance. The simulation result on this detector is illustrated in Figure 8. Through adjusting the focus lengths of the lenslets on the MLA the central hotspot is generated and the illuminance weakens along with the increasing degrees in both directions. The requirement of the high beam is achieved, in addition, a hotspot area of up to $\pm 2^{\circ}$ in horizontal direction can be realized. This hotspot area enables a digital cornering light, which provides a good field of view when driving around a corner. Moreover, the hotspot area includes the primary on-road projection range when driving straight on the way, which allows a high light and shade contrast of the projected content, leading to a noticeable lighting information for the driver and other road participants.

To shape the light in this way, the utilization factor can be increased from $11 \%$ to $39 \%$ with this concept for the considered light distribution, without the use of a distorting projection optics.

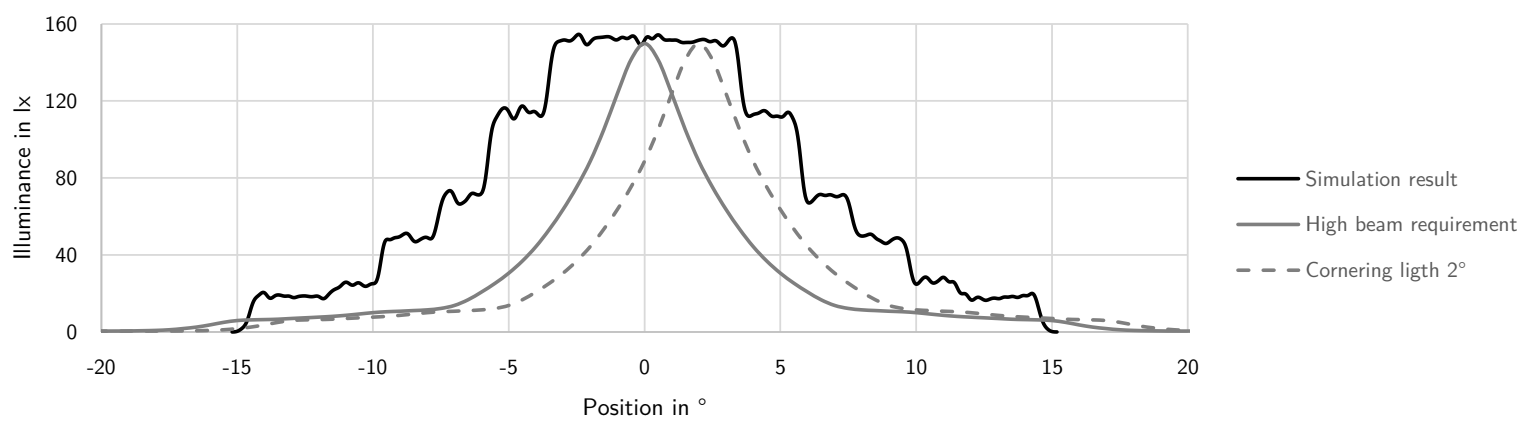

Figure 8. The simulated light distribution on a measuring wall in $25 \mathrm{~m}$ distance.

\section{CONCLUSION AND OUTLOOK}

In the beginning of this article the challenges of using area-based light modulators for high-resolution headlamp systems are discussed, followed by the distribution requirements for the road illumination of headlamp systems, the necessity of the central hotspot is also introduced. After that the system efficiency with respect to the optical efficiency and the utilization factor is illustrated, simultaneously with the efficiency promotion by the concept of inhomogeneous lighting. Whereafter a concept using the MLA to generate an inhomogeneous light distribution is introduced and validated by an simulation of the whole optical concept.

According to the simulation result in chapter 5, a central hotspot is realized on the area-based modulator's surface, then secondary optics are used to shape the light on both vertical and horizontal directions to realize the $\pm 15^{\circ}$ and $\pm 5^{\circ}$ distribution, which is the main illumination and on-road projection area. The simulation result meets the illumination requirements for headlamp systems, withal it also shows the potential to increase the optical efficiency and a to allow a cornering light.

\section{ACKNOWLEDGMENTS}

This project was funded by the Ministry of Science and Culture of Lower Saxony within the framework of project Tailored Light.

\section{REFERENCES}

[1] J. Moisel, "Requirements for future high resolution adb modules," Darmstädter Lichttechnik, 11th International Symposium on Automotive Lightning - ISAL 2015 - Proceedings of the Conference: Volume 16, Utz, Herbert, (München), 2015. 
[2] G. Kloppenburg, A. Wolf, and R. Lachmayer, "High-resolution vehicle headlamps: technologies and scanning prototype," Advanced Optical Technologies 5(2), pp. 147-155, 2016. DOI 10.1515/aot-2016-0001.

[3] M. Knöchelmann, G. Kloppenburg, and R. Lachmayer, "Headlamp innovations: optical concepts for fully adaptive light distributions," Proc.SPIE 10546, pp. 10546 - 10546 - 9, 2018. DOI 10.1117/12.2290013.

[4] M. Knöchelmann, A. Wolf, G. Kloppenburg, and R. Lachmayer, "Aktiver scheinwerfer mit dmd-technologie zur erzeugung vollstndiger lichtverteilungen," in 8. VDI-Tagung Optische Technologien in der Fahrzeugtechnik // Optische Technologien in der Fahrzeugtechnik, VDI, ed., VDI-Berichte 2323, pp. 61-78, VDI-Verl., (Düsseldorf), 2018. DOI: 10.15488/3975.

[5] Wirtschaftskommission für Europa der Vereinten Nationen, "Regelung nr. 113 der wirtschaftskommission für europa der vereinten nationen (unece) — einheitliche bedingungen für die genehmigung der kraftfahrzeugscheinwerfer für symmetrisches abblendlicht und/oder fernlicht, die mit glühlampen, gasentladungslichtquellen oder led-modulen ausgerüstet sind," 14.06.2014.

[6] C. Gut, I. Rotscholl, and C. Neumann, "Theoretische leistungs- und effizienzanalyse laserbasierter pixellichtsysteme," in 6. VDI-Tagung Optische Technologien in der Fahrzeugtechnik // Optische Technologien in der Fahrzeugtechnik, VDI, ed., VDI-Berichte 2221, pp. 15-29, VDI-Verl., (Düsseldorf), 2014.

[7] A. Günther, "Optical concept for an active headlamp with a dmd array," in Optical Sensors 2008, 7003, p. 70032D, International Society for Optics and Photonics, 2008.

[8] V. R. Bhakta and B. Ballard, "High resolution adaptive headlight using texas instruments dlp® technology," in Proceedings of the 11th International Symposium on Automotive Lighting], Khanh, TQ, ed, pp. 483-494, 2015.

[9] A. Wolf, G. Kloppenburg, R. Danov, and R. Lachmayer, "Dmd based automotive lighting unit," in $\mathrm{DGaO}$ proceedings 117. Jahrestagung, Deutsche Gesellschaft für angewandte Optik, ed., 2016. DOI: 10.15488/4004.

[10] A. Lizana, N. Martín, M. Estapé, E. Fernández, I. Moreno, A. Márquez, C. Iemmi, J. Campos, and M. Yzuel, "Influence of the incident angle in the performance of liquid crystal on silicon displays," $O p$ tics Express 17(10), pp. 8491-8505, 2009.

[11] Texas Instruments, DLP7000 DLP 0.7 XGA 2x LVDS Type A DMD, 11 2015. Rev. D. 